NOTICE: this is the author's version of a work that was accepted for publication in Applied Geochemistry. Changes resulting from the publishing process, such as peer review, editing, corrections, structural formatting, and other quality control mechanisms may not be reflected in this document. Changes may have been made to this work since it was submitted for publication. A definitive version was subsequently published in Applied Geochemistry, [VOL 55, (April 2015)] DOI 10.1016/j.apgeochem.2014.12.005•"

\title{
Use of redundant data to reduce estimation errors in geochemical speciation
}

\author{
F. De Gaspari ${ }^{\mathrm{a}, \mathrm{b}, *}$, M.W. Saaltink ${ }^{\mathrm{a}}$, J. Carrera ${ }^{\mathrm{b}}$, L.J. Slooten ${ }^{\mathrm{b}, \mathrm{c}}$ \\ ${ }^{a}$ GHS, Department of Geotechnical Engineering and Geosciences, Universitat Politecnica \\ de Catalunya, UPC-BarcelonaTech, c/Jordi Girona 1-3, 08034 Barcelona, Spain \\ ${ }^{b} G H S$, Institute of Enviromental Assessment and Water Research (IDEA), CSIC, \\ c/Jordi Girona 18, 08034 Barcelona, Spain \\ ${ }^{c}$ Alten Nederland, Rivium 1e straat 85, 2909 LE Capelle a/d IJssel, Nederland
}

\begin{abstract}
Speciation is the process of evaluating the concentrations of all the species in a chemical system from equilibrium conditions and measured data such as total concentrations of components, electrical conductivity, $\mathrm{pH}$, redox potential, gas partial pressure. It is essential for analyzing geochemical data and defining the chemical composition of waters for geochemical modeling problems like evaluating the chemical composition of evaporating, diluting, mixing waters or reactive transport. We present an algorithm that reduces estimation errors in chemical speciation calculations by means of the use of redundant data. Redundant data are measurements and assumptions that exceed the minimum data set required, and therefore are not strictly necessary, to speciate a water sample. The proposed method was compared with the classical speciation algorithm on two synthetic examples. Our re-

\footnotetext{
* Corresponding author

Email addresses: francesca.de.gaspari@upc.edu (F. De Gaspari), maarten.saaltink@upc.edu (M.W. Saaltink), jesus.carrera.ramirez@gmail.com (J. Carrera), luitjan.slooten@gmail.com (L.J. Slooten)
} 
sults show that using redundant data improves speciation results reducing the estimation error between computations and measurements. Moreover, the larger the amount of redundant data, the better in terms of errors of the estimated concentrations.

Keywords:

Redundant data, Geochemical modeling, Speciation, Optimization problem

\section{Introduction}

Geochemical modeling is important in Earth Sciences. In particular, it is 3 required to assess problems ranging from weathering to the characterization 4 of the chemical composition of water and processes that could influence its 5 quality (Appelo and Postma, 2010; Bethke, 2008). Geochemical speciation 6 is a key step of geochemical modelling that consists of evaluating concentra7 tions of all the species in a chemical system from measured data (e.g., total 8 concentrations of components, $\mathrm{pH}$, alkalinity, gas partial pressures, electrical 9 conductivity, redox potential) and equilibrium constraints. For this reason, 10 it is often termed thermodynamic speciation.

Speciation requires the solution of a non-linear system of equations and 12 a lot of research has been focused on numerical issues that might arise when solving these equations. Several methods have been proposed to solve chem-

García et al., 2013; Carrayrou et al., 2002; Brassard and Bodurtha, 2000) and many codes have also been released to deal with geochemical speciation calculations: GEMS3K (Kulik et al., 2013), Visual MINTEQ (Gustafsson, 2011), CHEPROO (Bea et al., 2009), ORCHESTRA (Meeussen, 2003), 
MIN3P (Mayer et al., 2002), PHREEQC (Parkhurst et al., 1999) and its interactive version, PHREEQCi (Charlton et al., 1997), EQ3NR (Wolery, 1983, 1992) and WATEQ4F (Ball and Nordstrom, 1991).

Speciation calculations are subject to implicit sources of uncertainty which can derive from uncertainty in thermodynamic data, such as equilibrium constant values, or from errors in chemical analyses (i.e., analytical errors). These types of random errors can be referred to as "aleatory uncertainty". Misjudgment in the definition of the chemical system, such as failure to account for some reactions or discarding others, can also lead to errors in speciation. These can be defined as "epistemic uncertainty". They arise from an incomplete or inadequate characterization of the system (Gupta et al., 2012), such as assuming the neutrality of a solution when it is not electrically balanced, or imposing equilibrium with phases that are not. The effect of errors propagation in geochemical calculations has been extensively studied. In particular, the effect of aleatory errors has been investigated by Weber et al. (2006); Denison and Garnier-Laplace (2005); Ödegaard-Jensen et al. (2004); Nitzsche et al. (2000); Cabaniss (1999, 1997); Criscenti et al. (1996); Merino (1979), while Smith et al. (1999) examined the connection between aleatory and epistemic errors. Although the origin and propagation effects of both types of errors are different, they can be treated in the same way through probability density functions, e.g., by means of mean and standard deviation values.

All these studies use a fixed number of data to solve the speciation. Geochemical speciation, in fact, requires a fixed minimum number of data, including equilibrium assumptions, equal to the number of independent variables 
of the system (i.e., number of species). For example, a carbonate system is characterized by four degrees of freedom (see Section 2.1). Therefore, four data (e.g., total concentrations of inorganic carbon and calcium, $p H$ ) or hypotheses (e.g., water activity equal to 1) are needed. However, extra data might be available (e.g., alkalinity, electrical conductivity or redox potential) or extra assumptions about the system might be made (e.g., equilibrium with calcite or $\mathrm{CO}_{2(g)}$ in equilibrium with the atmosphere). Chemical analyses of waters, for example, often provide extra data and also the analytical errors associated to each of them.

We term these extra data as redundant and we claim that speciation calculations can benefit from their use, while aknowledging analytical errors.

The aim of this paper is to present an algorithm to include redundant data in speciation calculations and to prove that their use can improve the results by reducing estimation errors. We also claim that increasing the number of redundant data helps decreasing the estimation errors even further.

\section{Methodology}

We start by analyzing a speciation example to clarify the differences between the traditional and the proposed method. This allows us to formalize the problem statement and to propose a solution algorithm.

\subsection{Speciation of a carbonate system}

We consider the problem of calculating the concentrations of dissolved species in a carbonate system. This system has received extensive attention from the scientific community, e.g. to study seawater intrusion in carbonate coastal aquifers (Werner et al., 2013; Bear, 1999; Back et al., 1979, amongst 
many others), including geochemical processes occurring in the mixing zone between freshwater and saltwater (Sanz et al., 2011; De Simoni et al., 2007; Rezaei et al., 2005), and to analyze the feasibility of $\mathrm{CO}_{2}$ sequestration in deep aquifers (Saaltink et al., 2013; Duan and Li, 2008; Xu et al., 2006).

The most simple chemical system consists of 9 species $\left(N_{s}=9\right)$ and the following 5 equilibrium reactions $\left(N_{r e}=5\right)$ :

$$
\begin{array}{ll}
\mathrm{OH}^{-}+\mathrm{H}^{+} \rightleftharpoons \mathrm{H}_{2} \mathrm{O} & \log K_{1}=13.995 \\
\mathrm{CO}_{3}^{2-}+\mathrm{H}^{+} \rightleftharpoons \mathrm{HCO}_{3}^{-} & \log K_{2}=10.328 \\
\mathrm{CO}_{2(a q)}+\mathrm{H}_{2} \mathrm{O} \rightleftharpoons \mathrm{HCO}_{3}^{-}+H^{+} & \log K_{3}=-6.344 \\
\mathrm{CO}_{2(g)}+\mathrm{H}_{2} \mathrm{O} \rightleftharpoons \mathrm{HCO}_{3}^{-}+H^{+} & \log K_{4}=-7.813 \\
\mathrm{CaCO}_{3(s)}+\mathrm{H}^{+} \rightleftharpoons \mathrm{Ca}^{2+}+\mathrm{HCO}_{3}^{-} & \log K_{5}=1.848
\end{array}
$$

The number of degrees of freedom of this system is $N_{s}-N_{r e}=4$. This means that 4 data or assumptions are needed to solve the speciation problem. Speciation codes normally use this criterion. Optionally species with constant activity can be decoupled and eliminated, e.g., water if the system is sufficiently diluted $\left(\mathrm{a}_{H_{2} \mathrm{O}}=1\right)$ or proton if the $p H$ is fixed $\left(\mathrm{a}_{H^{+}}=10^{-p H}\right)$, to reduce the number of unknowns. Numerous methods have been proposed to eliminate constant activity species in reactive transport calculations (Kräutle, 2011; De Simoni et al., 2005; Kräutle and Knabner, 2005; Molins et al., 2004; Saaltink et al., 1998). Regardless of the decision to eliminate them, we refer generically to these methods as the traditional speciation methods, as they should all yield the same results.

Being the degrees of freedom for system (1) equal to 4 , the concentrations 
of all species can be calculated from four known data: total concentration of 87 calcium, alkalinity, activity of water and $p H$ for example

$$
\left\{\begin{array}{l}
C a_{\text {tot }}:\left[\mathrm{Ca}^{2+}\right]-x_{1}=0 \\
\text { Alkalinity }:\left[\mathrm{HCO}_{3}^{-}\right]+2\left[\mathrm{CO}_{3}^{2-}\right]+\left[\mathrm{OH}^{-}\right]-\left[\mathrm{H}^{+}\right]-x_{2}=0 \\
\text { Water Activity }: \mathrm{a}_{H_{2} O}-x_{3}=0 \\
p H:-\log \mathrm{a}_{H^{+}}+x_{4}=0
\end{array}\right.
$$

88 where [] represents molal concentration (mol $/ \mathrm{kgw}) . x_{1}, x_{2}$ and $x_{4}$ are 89 actual measurements representing $C a_{t o t}$, Alkalinity and $p H$, while $x_{3}$ is the value of water activity fixed to 1 . We term these kind of equations "data equations". These must be solved together with the mass action laws deriving 92 from system (1)

$$
\mathbf{f}_{M A L}=\mathbf{S}_{e} \log \mathbf{a}-\log \mathbf{k}=0
$$

93

96

where $\mathbf{a}$ is a vector containing the activities of the $N_{s}$ species, $\mathbf{S}_{e}$ is a matrix $\left(N_{r e} \times N_{s}\right)$ with the stoichiometric coefficients of the equilibrium reactions and $\mathbf{k}$ is a vector $\left(N_{r e}\right)$ of equilibrium constants.

Generalizing the traditional speciation method we can say that $N_{1}=$ $N_{s}-N_{r e}$ data equations need to be solved together with $N_{2}=N_{r e}$ mass action laws, $\mathbf{f}_{M A L}$ :

$$
\left\{\begin{array}{l}
\mathbf{g}(\mathbf{c})-\mathbf{x}=0 \\
\mathbf{f}_{M A L}(\mathbf{c})=0
\end{array}\right.
$$

where $\mathbf{c}$ is the vector of concentrations of the $N_{s}$ species, $\mathbf{x}$ a vector of $N_{1}$ data and $\mathbf{g}(\mathbf{c})$ defines operations to be applied to $\mathbf{c}$ in order to compute what 
is measured (e.g., linear combinations of species concentrations to obtain measured components, or $-\log \left(\gamma_{H^{+}} \cdot\left[H^{+}\right]\right)$to obtain $p H$, where $\gamma_{H^{+}}$is the proton activity coefficient). Typically data equations contain balances of total concentrations, electrical charge, alkalinity, total dissolved inorganic carbon $(T I C), p H$ values, redox potential or electrical conductivity.

The traditional algorithm to speciate consists of five steps: (1) dividing the species in two sets of $N_{1}=N_{s}-N_{r e}$ primary and $N_{2}=N_{r e}$ secondary species (Steefel and Yabusaki, 2000) with concentrations $\mathbf{c}_{1}$ and $\mathbf{c}_{2}$, respectively; (2) guess an initial value of primary concentrations; (3) use $\mathbf{f}_{M A L}$ to calculate $\mathbf{c}_{2}=f\left(\mathbf{c}_{1}\right)$; (4) use data $\mathbf{x}$ to solve $g\left(\mathbf{c}_{1}, \mathbf{c}_{2}\right)-\mathbf{x}=0$ for $\mathbf{c}_{1}$, (5) repeat steps (3) and (4) until convergence.

This work is focused on cases in which the number of available data is larger than $N_{1}$. In this case, the resulting data equations cannot be solved exactly. Instead, they need to aknowledge measurement errors.

For example, if measurements of total dissolved inorganic carbon $(T I C)$ and pressure of gas $\left(P_{C O_{2(g)}}\right)$ were available and we wanted to apply zero charge balance and equilibrium with calcite as well, the data equations could be rewritten as 


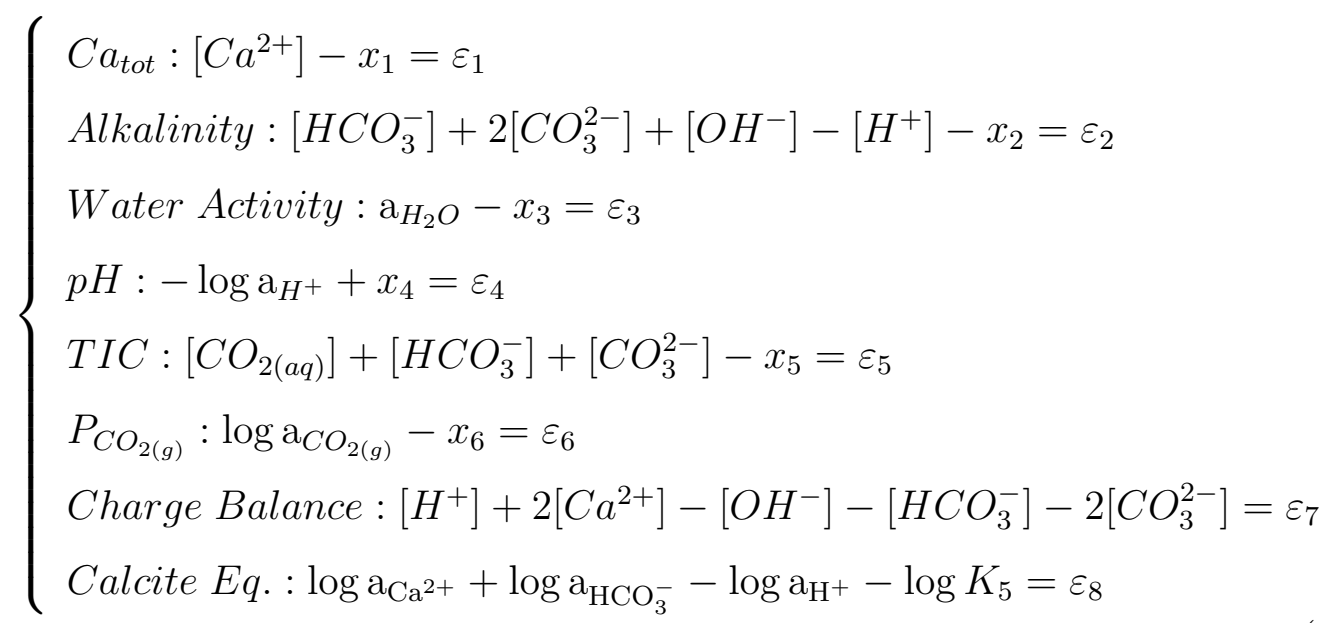

where $x_{5}$ is the measured TIC, $x_{6}$ is $\log \left(P_{\left.C O_{2(g)}\right)}\right)$ and $x_{7}$ and $x_{8}$ are equal to zero because of the zero charge balance and equilibrium constraints ( $x_{7}$ corresponds to the saturation index of calcite, null at equilibrium). $\varepsilon_{i}$, $i=1, \ldots, 8$, represent measurement errors that need to be taken in account since the system to be solved has become overdetermined (i.e., the number of data is larger than $N_{1}$ ). The data set (5) presents 4 redundant data.

The algorithm to solve data equations (5) together with mass action laws (3) to speciate is explained in the following section.

\subsection{Speciation with redundant data: Problem statement}

If redundant informations are used to solve a speciation problem, system (4) can be re-defined as follows

$$
\left\{\begin{array}{l}
\mathbf{g}(\mathbf{c})-\mathbf{x}=\boldsymbol{\varepsilon} \\
\mathbf{f}_{M A L}(\mathbf{c})=0
\end{array}\right.
$$


The differences of system (6) from the traditional speciation problem defined in (4) are the dimension of $\mathbf{g}$ and $\mathbf{x}\left(\operatorname{dim}(\mathbf{g})=\operatorname{dim}(\mathbf{x})=N_{d}>\right.$ $N_{1}$ ) and errors in measurements $\varepsilon$ which are included. $\varepsilon$ can incorporate analytical errors in data, such as in data 1 to 4 in system (5), and uncertainty about the correct model, such as charge balance and equilibrium with calcite assumptions in system (5).

When solving speciation problems, it is common to use data equations which are either linear combinations of concentrations (e.g., TIC, alkalinity) or linear combinations of log-activities (e.g., equilibrium with minerals or gases). Moreover, the errors $(\varepsilon)$ of both types of data equations can have a normal or log-normal distribution. Therefore, the expressions of $\mathbf{g}(\mathbf{c})$ must be defined and calculated accordingly to the types of data equations (see Appendix A for details).

System (6) is overdetermined, therefore a non-linear least square fitting is required to minimize $\varepsilon$, as described below.

\subsection{Algorithm}

We want to find the solution of (6) that minimizes the sum $S$ of the weighted squares of the difference between measured and calculated data, defined as

$$
S=\varepsilon^{t} \mathbf{V}^{-1} \boldsymbol{\varepsilon}
$$

where $\mathbf{V}$ is the covariance matrix $\left(N_{d} \times N_{d}\right)$ of measurement errors. Without loss of generality, we will assume errors to be not correlated, so that $\mathbf{V}$ is a diagonal matrix, containing the variance of each $i$-th measurement, $\sigma_{e, i}^{2}$. 
with

$$
\Delta \ln \mathbf{c}_{1}^{k}=\ln \mathbf{c}_{1}^{k+1}-\ln \mathbf{c}_{1}^{k}
$$
and substituting it in (8), the solution for a given iteration $k$ is

$$
\mathbf{J}^{t} \mathbf{V}^{-1} \mathbf{J} \Delta \ln \mathbf{c}_{1}=-\mathbf{J}^{t} \mathbf{V}^{-1} \boldsymbol{\varepsilon}
$$

$$
\frac{\partial S}{\partial \ln \mathbf{c}_{1}}=2 \varepsilon^{t} \mathbf{V}^{-1} \frac{\partial \mathbf{g}}{\partial \ln \mathbf{c}_{1}}=2 \varepsilon^{t} \mathbf{V}^{-1} \mathbf{J}=\mathbf{0}
$$

$$
\varepsilon^{k+1}=\varepsilon^{k}+\mathbf{J}^{k} \Delta \ln \mathbf{c}_{1}^{k}
$$

\footnotetext{
After convergence, the covariance matrices of the estimation errors associated to $\ln \mathbf{c}_{1}\left(\boldsymbol{\Sigma}_{1}\right)$ and $\ln \mathbf{c}_{2}\left(\boldsymbol{\Sigma}_{2}\right)$ can be calculated. This can be useful to analyze the quality of the estimation (see section 2.4). $\Sigma_{1}$ and $\Sigma_{2}$, in fact, provide the uncertainty associated to the estimation of $\ln \mathbf{c}_{1}$ and $\ln \mathbf{c}_{2} . \boldsymbol{\Sigma}_{1}$ $\left(N_{1} \times N_{1}\right)$ can be calculated by means of the "real" covariance matrix of the data, $\mathbf{C}_{d}\left(N_{d} \times N_{d}\right)$ :
} 


$$
\boldsymbol{\Sigma}_{1}=\operatorname{Cov}\left(\ln \mathbf{c}_{1}\right)=\left(\mathbf{J}^{t} \mathbf{C}_{d}^{-1} \mathbf{J}\right)^{-1}
$$

However, in reality $\mathbf{C}_{d}$ is not known. Therefore, it is necessary to make an hypothesis about its structure. A reasonable assumption defines $\mathbf{C}_{d}$ as proportional to $S$ and to the covariance of measurement errors, $\mathbf{V}$ :

$$
\mathbf{C}_{d}=\sigma^{2} \mathbf{V}
$$

where $\sigma^{2}=S / N_{d}$ (S was defined in equation 7). Substituting (13) into (12) we obtain

$$
\boldsymbol{\Sigma}_{1}=\sigma^{2}\left(\mathbf{J}^{t} \mathbf{V}^{-1} \mathbf{J}\right)^{-1}
$$

$\boldsymbol{\Sigma}_{2}\left(N_{2} \times N_{2}\right)$ can be calculated by taking into account the dependence of $\mathbf{c}_{2}$ on $\mathbf{c}_{1}$

$$
\boldsymbol{\Sigma}_{2}=\operatorname{Cov}\left(\ln \mathbf{c}_{2}\right)=\left(\frac{\partial \ln \mathbf{c}_{2}}{\partial \ln \mathbf{c}_{1}}\right) \boldsymbol{\Sigma}_{1}\left(\frac{\partial \ln \mathbf{c}_{2}}{\partial \ln \mathbf{c}_{1}}\right)^{t}
$$

Details on the calculation of $\left(\partial \ln \mathbf{c}_{2} / \partial \ln \mathbf{c}_{1}\right)$ are explained in Appendix B.

The steps of the proposed algorithm can be outlined as follows:

1. Set $\mathbf{x}$ and matrices of $\mathbf{g}(\mathbf{c})$ (see Appendix A)

2. Guess initial value of $\mathbf{c}_{1}^{0}$

3. Given $\mathbf{c}_{1}^{k}$, calculate secondary concentrations $\mathbf{c}_{2}^{k}=f\left(\mathbf{c}_{1}^{k}\right)$ and $\partial \mathbf{c}_{2}^{k} / \partial \mathbf{c}_{1}^{k}$ from $\mathbf{f}_{M A L}=0$ (see Appendix B) 
4. Calculate $\boldsymbol{\varepsilon}^{k}$, Jacobian matrix $\mathbf{J}^{k}$, and RHS and LHS of system (11). Solve system (11) and evaluate $\Delta \ln \mathbf{c}_{1}^{k}$

5. Update the solution $\ln \mathbf{c}_{1}^{k+1}=\ln \mathbf{c}_{1}^{k}+\Delta \ln \mathbf{c}_{1}^{k}$

6. Set $\mathrm{k}=\mathrm{k}+1$ and repeat steps 3 . to 5 . until convergence

7. After convergence, calculate $\boldsymbol{\Sigma}_{1}$ and $\boldsymbol{\Sigma}_{2}$

As convergence criteria, we check the maximum relative error between two sequential iterations and the residual (RHS in system 11) at every iterations. The iterative process is stopped when both quantities are smaller than threshold values defined by the user.

\subsection{Testing approach}

The algorithm was tested by means of two synthetic examples: first a single reaction representing gypsum equilibrium at a temperature of $25^{\circ} \mathrm{C}$ in ideal conditions $(\mathbf{a}=\mathbf{c})$

$$
\mathrm{CaSO}_{4(s)} \rightleftharpoons \mathrm{Ca}^{2+}+\mathrm{SO}_{4}^{2-} \quad \log \mathrm{K}=-4.482
$$

We chose arbitrarly a known solution of (16) and 5 possible measurements with errors of $\log \left[\mathrm{Ca}^{2+}\right]$ and $\log \left[\mathrm{SO}_{4}^{2-}\right]$, decimal logarithm of calcium and sulfate concentrations, respectively. Figure 1 shows the equilibrium line and the exact solution of the speciation together with the 5 measurement points that were used in this case. Both the traditional and the proposed methods were applied to solve the speciation. Since the system is characterized by one degree of freedom, i.e. one datum is necessary to solve the speciation, first the traditional method was employed using only $\log \left[\mathrm{Ca}^{2+}\right]$ data, and 
later the proposed method was employed using both $\log \left[\mathrm{Ca}^{2+}\right]$ and $\log \left[\mathrm{SO}_{4}^{2-}\right]$ measurements. The results were first compared in terms of logaritmic mean squared error, $M S E_{\text {log }}$ :

$$
M S E_{l o g}=\frac{1}{N} \frac{1}{N_{s}} \sum_{i=1}^{N} \sum_{j=1}^{N_{s}}\left[\log \left(\frac{c_{i j}}{c_{j}^{*}}\right)\right]^{2}
$$

where $N$ is the number of measurements (5), $N_{s}$ is the number of species (2), $c_{i j}$ is the calculated concentration of the $i$-th measurement and $j$-th species, and $c_{j}^{*}$ is the exact value of the $j$-th species. Comparing the two methods in terms of $M S E_{l o g}$ was possible because the exact solution in this case is known. However, when a speciation is calculated using real data the exact solution is not known a priori. Therefore, we computed the covariance matrices of estimation errors $\boldsymbol{\Sigma}_{1}$ and $\boldsymbol{\Sigma}_{2}$ defined in (14) and (15), respectively, and compared the estimation errors of the traditional and the proposed methods also in terms of the variable $\operatorname{Var}^{*}$ :

$$
\operatorname{Var}^{*}=\frac{1}{N} \frac{1}{N_{s}} \sum_{i=1}^{N}\left[\sum_{l=1}^{N_{1}}\left(\Sigma_{1, l l}\right)_{i}+\sum_{m=1}^{N_{2}}\left(\Sigma_{2, m m}\right)_{i}\right]
$$

$V a r^{*}$ represents a mean value of the estimation errors variance of all the species. From (14) it can be noticed that $\boldsymbol{\Sigma}_{1}$ depends on the error $\varepsilon$ through the variable $\sigma^{2}$. However, for the traditional speciation method $\varepsilon=0$, hence $S=0 \Rightarrow \sigma^{2}=0$ (see system 4). This makes (14) not suitable for the comparison. Therefore, we fixed $\sigma^{2}=1$ for the two methods. This way, we still guarantee that the real covariance matrix of data, $\mathbf{C}_{d}$ (see equation 13), is the same for both methods.

An input variable of the method is the variance of measurement errors 
associated to the data, $\sigma_{e}^{2}$ (see equation 7 ). In this example we used the same value for both $\log \left[\mathrm{Ca}^{2+}\right]$ and $\log \left[\mathrm{SO}_{4}^{2-}\right]$ data $\left(\sigma_{e}=0.17\right)$, so that they have the same weight. In order to make the results of this example more general we tested again the two methods for 1500 measurements of $\log \left[\mathrm{Ca}^{2+}\right]$ and $\log \left[S O_{4}^{2-}\right]$ with $\sigma_{e}=0.17$. The exact solution was therefore perturbed 1500 times by means of a lognormal distribution with standard deviation $\sigma_{g}=0.17$ to obtain the measurement points shown in figure 2 . The use of a lognormal distribution allowed us to avoid possible negative values in concentrations in the measurements generation process.

The advantage of this simple problem is that it presents an analytical solution. In the case of using only $\log \left[\mathrm{Ca}^{2+}\right]$ data, the variance of $\log \left[\mathrm{Ca}^{2+}\right]$ error will be $\sigma_{e}^{2}$ because $\log \left[\mathrm{Ca}^{2+}\right]$ will remain unchanged. The same error will transfer to $\log \left[\mathrm{SO}_{4}^{2-}\right]=\log K-\log \left[\mathrm{Ca}^{2+}\right]$. Therefore, the expected value of $\operatorname{Var}^{*}$ is $\sigma_{e}^{2}$. If both $\log \left[\mathrm{Ca}^{2+}\right]$ and $\log \left[\mathrm{SO}_{4}^{2-}\right]$ measurements are used, it is easy to check that minimizing $\varepsilon_{\mathrm{Ca}^{2+}}^{2}+\varepsilon_{\mathrm{SO}_{4}^{2-}}^{2}$, where $\varepsilon_{i}=\log c_{i}-x_{i}(i=$ $\left.\mathrm{Ca}^{2+}, \mathrm{SO}_{4}^{2-}\right)$, subject to $\log \left[\mathrm{Ca}^{2+}\right]+\log \left[\mathrm{SO}_{4}^{2-}\right]=\log \mathrm{K}$, leads to $\log \left[\mathrm{Ca}^{2+}\right]=$ $\left(\log K+x_{C a^{2+}}-x_{\mathrm{SO}_{4}^{2-}}\right) / 2$. Thus, the variance of both estimates is $\sigma_{e}^{2} / 2$, which is the expected value of $\operatorname{Var}^{*}$. This result will serve to test our approach and to illustrate the advantage of using redundant data.

As second example we chose the carbonate system defined in (1). The extended Debye-Hückell expression for activity coefficients was used in this example (Helgeson and Kirkham, 1974). As explained in section (2.1) the system is characterized by four degrees of freedom. We used as exact solution a water in equilibrium with calcite, with partial pressure of $\mathrm{CO}_{2(g)}$ equal to $10^{-3.5}, \mathrm{a}_{\mathrm{H}_{2} \mathrm{O}}=1$ and electrically balanced. Its chemical composition is shown 
in Table 1.

First we compared the traditional and the proposed methods to verify the accuracy of the two algorithms in terms of speciation results. For this purpose, six data were extracted from the exact solution to be used in the speciation calculations (Table 2). The traditional speciation method was employed using four data equations: alkalinity, $C a_{t o t}, \mathrm{a}_{\mathrm{H}^{+}}$and $\mathrm{a}_{\mathrm{H}_{2} \mathrm{O}}=1$. Note that the proton activity presents a measurement error, therefore cannot be eliminated. We will refer to this case as 'solution 1'. Afterwards we tested the proposed method adding gradually redundant data to the previous three: TIC ('solution 2'), activity of the gas, $\mathrm{a}_{\mathrm{CO}_{2(g)}}$ ('solution 3') and equilibrium with calcite condition ('solution 4'). Afterwards we compared the solution obtained with the traditional speciation method to the solutions obtained using an increasing number of redundant data: from 1 in solution 2 to 3 in solution 4. As for the gypsum example, we perturbed the data to generate 1500 possible measured values and then we compared the speciation results in terms of $M S E_{l o g}$ and $V a r^{*}$, defined in (17) and (18), respectively.

The measured values were generated perturbing the logarithm of the exact value $(\mu)$ with a standard deviation, $\sigma_{g}$, of 0.17 by means of a log-normal distribution. The condition of calcite equilibrium was not perturbed $\left(\sigma_{g}=0\right)$, since zero is the reference value of the saturation index for minerals in equilibrium. The values of $\sigma_{g}$ were used also to define the uncertainty associated to every datum presenting analytical errors $\left(\sigma_{e}=0.17\right.$, see Table 2$)$.

In reality, however, it is difficult to know the correct value of uncertainty for each type of measurement. To analyze the effect of an incorrect measurement error we performed a second group of simulations in which we changed 
the $\sigma_{e}$ values of all data one at a time and calculated $M S E_{l o g}$ and $\operatorname{Var}^{*}$ as function of the standard deviation of measurement errors of every constraint. We chose two values of $\sigma_{e}$ : the first larger than the one used to generate the perturbed measurements $\left(\sigma_{e}=0.35>\sigma_{g}\right)$, to simulate a higher uncertainty associated to the data, and the second smaller $\left(\sigma_{e}=0.09<\sigma_{g}\right)$, to simulate more certain data values.

\section{Results}

\subsection{Gypsum example}

Figure 3 shows the results of the traditional speciation method, i.e. using only $\log \left[\mathrm{Ca}^{2+}\right]$ data. It can be noticed that the five points moved on the equilibrium line, since the equilibrium with gypsum was imposed as certain condition, along a line parallel to the y-axis which represents the imposed calcium concentration data. In this case $M S E_{\text {log }}=0.28$ and $\operatorname{Var}^{*}=0.154$. Note that $\operatorname{Var}^{*}$ coincides with its expected value, $\sigma_{e}^{2}$, once $\ln$ is converted to $\log _{10}$. Afterwards, the proposed method was tested, i.e., using both $\log \left[\mathrm{Ca}^{2+}\right]$ and $\log \left[\mathrm{SO}_{4}^{2-}\right]$ data. The results are shown in figure 4 . It can be observed that while some of the points moved further from the exact solution with respect to the classical speciation results (white and black triangles), the others moved closer to the exact solution. However, for all the points the proposed algorithm minimizes the distance between measured and calculated data. The calculated mean squared error in this case was 0.23 , smaller than 0.28 obtained with the traditional method. Moreover, $\operatorname{Var}^{*}=0.077$, which coincides again with its expected value, $\sigma_{e}^{2} / 2$.

The same methodology was employed to compare the two methods for 
the 1500 measurement points of figure 2 and the resulting mean squared error decreased from 0.029 for the traditional method to 0.016 for the proposed methods. The value of $\operatorname{Var}^{*}$ also decreased by half, confirming its expected value: from 0.156 to 0.078 for traditional and proposed methods, respectively.

\subsection{Carbonate example}

The results of the comparison between the two methods in terms of $M S E_{\log }$ are shown in figure 5 . It can be noticed that the value of the mean squared error for the traditional method (solution 1) is barely larger than 0.05, while it is smaller for the solutions using redundant data (solutions 2, 3 and 4). Moreover, increasing the number of redundant data used in the speciation contributes to reduce more the $M S E_{l o g}$ value: it decreases from 0.04 using only one redundant data (solution 2) to 0.016 using 3 redundant data (solution 4).

Figure 6 shows the effect of changing the standard deviation associated to measurements $\left(\sigma_{e}\right)$. Obviously its value does not affect solution 1, which is the result of a traditional speciation calculation. Neither does the value of $\sigma_{e}$ for $\mathrm{a}_{C O_{2(g)}}$ have an effect on solution 2 (figure 6e) because this solution does not use $\mathrm{a}_{C O_{2(g)}}$ data. Nor does the $\sigma_{e}$ for the assumption of equilibrium with calcite have an effect on solutions 2 and 3 (figure $6 \mathrm{f}$ ), for the same reason. In general one can observe that the use of an incorrect standard deviation can worsen the solution with respect to the one obtained with the correct standard deviations. However, the quality of the solution in terms of $M S E_{l o g}$ improves using redundant data with respect to the traditional speciation, despite a wrong choice about $\sigma_{e}$ value. Decreasing the uncertainty relative to the equilibrium with calcite assumption (figure 6f) improves the 
solution even with respect to the one obtained with correct $\sigma_{e}$ values. This is because the correct value of $\sigma_{g}$ for this datum is 0 , not 0.17 (see Table 2). The effect of $\sigma_{e}$ relative to alkalinity and TIC (figure 6c, d) are very similar as their values are very close, due to the fact that in this $p H$ range the concentration of $\mathrm{HCO}_{3}^{-}$is predominant with respect to carbonate species or $\mathrm{OH}^{-}$concentrations. Finally, it seems that changing the uncertainty relative to $C a_{t o t}$ (figure 6a) does not affect the solution. Nevertheless, when a large number of redundant data is used, such as in solution 4, the standard deviation seems to have a minor effect on the estimation error, $M S E_{l o g}$.

The effect of an incorrect value of $\sigma_{e}$ on $\operatorname{Var}{ }^{*}$ was also analyzed. Only the results for $\mathrm{a}_{H^{+}}$and alkalinity are reported in figure 7 as the most representative. It can be noticed that the error variance can be big for the traditional speciation method (solution 1), while it slightly decreases when redundant data are used (solutions 2, 3 and 4). Moreover, the more redundant data are used, the more the variance of estimation error decreases, converging to a value corresponding to the true standard deviation of measurement errors $\left(\sigma_{e}=0.17\right)$

\section{Conclusions}

We proposed a speciation algorithm that uses redundant data and aknowledges measurement errors, on the assumption that redundant data will reduce estimation errors in geochemical calculations.

We compared the proposed method with the traditional speciation method in terms of logaritmic mean squared error, $M S E_{l o g}$ and mean value of estimation error variance, $\operatorname{Var}^{*}$. We tested both algorithms by means of two 
synthetic examples. Both $M S E_{l o g}$ and $V_{a r}^{*}$ using redundant data are consistently smaller than in the traditional method.

The effect of measurement errors was examined in a carbonate system example. The algorithm is sensitive to the variance of measurement errors. Also, a wrong value of the standard deviation can worsen the results with respect to the ones obtained with the correct standard deviation. However, the effect of its value depends on the type of data associated to it. A wrong error associated to measurements can still improve the results in terms of mean squared error and variance of estimation error with respect to a traditional speciation method, especially when a large number of redundant data are used.

Therefore we argue that the proposed method can improve the quality of the speciation results, reducing estimation errors.

\section{Appendix A. Error definitions}

The errors $\varepsilon$ allowed in the proposed method can be additive or multiplicative. Additive errors should lead to gaussian distributions, whereas multiplicative to lognormal distributions. Depending on the type of error, the function $\mathbf{g}(\mathbf{c})$ and the data $\mathbf{x}$ must be defined accordingly: aritmetic or logaritmic for additive and multiplicative errors, respectively.

Data used in speciation calculations are typically combinations of concentrations or activity values. The former, which we name balance equations, are linear combinations of concentrations representing total concentrations, alkalinity, charge balance or TIC values. The latter are usually employed to fix $\mathrm{pH}$ values or to impose equilibrium with minerals or gases. 


$$
\varepsilon=\mathrm{Bc}-\mathrm{x}
$$

371 for the balance equations and

$$
\varepsilon_{i}=\prod_{n=1}^{N_{s}} a_{n}^{L_{i n}}-x_{i}
$$

372 for each $i-t h$ activity combination, respectively. $\mathbf{B}$ is a matrix of di373 mension $\left(N_{b} \times N_{s}\right)$ and $N_{b}$ is the number of balance equations. B contains different coefficients depending on the type of balance equation: ionic charge for charge neutrality, coefficients defining alkalinity or TIC, or component matrix elements for total concentration. $\mathbf{L}$ is a matrix of dimension $\left(N_{a} \times N_{s}\right)$ containing the coefficients of the activity for every species involved in the combination. $N_{a}$ is the number of activity conditions imposed.

If we want to use a log-distribution instead of a normal distribution of errors, one should use:

$$
\varepsilon=\ln (\mathbf{B c})-\ln \mathbf{x}
$$

381 for the balance equations and

$$
\varepsilon=\mathbf{L} \ln \mathbf{a}-\ln \mathbf{x}
$$

382 for the activity combinations, respectively. 
383

384 385

\section{Appendix B. Jacobian calculation}

The jacobian, containining the derivatives of $\varepsilon$ with respect to the state variables $\ln \mathbf{c}_{1}$ at every step of the iterative method, can be calculated as

$$
\begin{gathered}
\frac{\partial \varepsilon_{i}}{\partial \ln c_{1, j}}=\frac{\partial \varepsilon_{i}}{\partial c_{1, j}} \cdot c_{1, j} \\
=\mathrm{B}_{1, i j} \cdot c_{1, j}+\sum_{l=1}^{N_{2}} \mathrm{~B}_{2, i l} \cdot \frac{\partial c_{2, l}}{\partial c_{1, j}} \cdot c_{1, j} \\
i=1, \ldots, N_{b} \\
j=1, \ldots, N_{1}
\end{gathered}
$$

$$
\begin{aligned}
\frac{\partial \varepsilon_{i}}{\partial \ln c_{1, j}}= & \frac{\partial \ln z_{i}}{\partial \ln c_{1, j}} \\
= & \frac{1}{z_{i}} \cdot \frac{\partial z_{i}}{\partial \ln c_{1, j}} \\
= & \frac{1}{z_{i}} \cdot\left(\mathrm{B}_{1, i j} \cdot c_{1, j}+\sum_{l=1}^{N_{2}} \mathrm{~B}_{2, i l} \cdot \frac{\partial c_{2, l}}{\partial c_{1, j}} \cdot c_{1, j}\right) \\
& \quad i=1, \ldots, N_{b} \\
& j=1, \ldots, N_{1}
\end{aligned}
$$

from the definition (19) whereas by means of definition (21) results

being

$$
z_{i}=\left(\sum_{m=1}^{N_{1}} \mathrm{~B}_{1, i m} \cdot c_{1, m}+\sum_{l=1}^{N_{2}} \mathrm{~B}_{2, i l} \cdot \frac{\partial c_{2, l}}{\partial c_{1, j}} \cdot c_{1, j}\right)
$$

Matrices $\mathbf{B}_{1}$ and $\mathbf{B}_{2}$ are the parts of matrix $\mathbf{B}$ relative to primary and secondary species, respectively, and the derivatives of secondary concentrations with respect to primary concentrations can be calculated considering 
respect to primary species concentrations is null:

$$
\frac{d \mathbf{f}_{M A L}}{d \ln \mathbf{c}_{1}}=\frac{\partial \mathbf{f}_{M A L}}{\partial \ln \mathbf{c}_{1}}+\frac{\partial \mathbf{f}_{M A L}}{\partial \ln \mathbf{c}_{2}} \frac{\partial \ln \mathbf{c}_{2}}{\partial \ln \mathbf{c}_{1}}=0
$$

393

Those derivative can be calculated by means of the following linear system

$$
\frac{\partial \mathbf{f}_{M A L}}{\partial \ln \mathbf{c}_{2}} \frac{\partial \ln \mathbf{c}_{2}}{\partial \ln \mathbf{c}_{1}}=-\frac{\partial \mathbf{f}_{M A L}}{\partial \ln \mathbf{c}_{1}}
$$

${ }_{394}$ The conversion to $\partial \mathbf{c}_{2} / \partial \mathbf{c}_{1}$ is straightforward, recalling that $d \ln x / d x=$ $3951 / x:$

$$
\frac{\partial c_{2, i}}{\partial c_{1, j}}=\frac{c_{2, i}}{c_{1, j}} \frac{\partial \ln c_{2, i}}{\partial \ln c_{1, j}}
$$

396 The derivatives of (22), remembering the definition of activity $(\mathbf{a}=\boldsymbol{\gamma} \cdot \mathbf{c})$ 397 and that $\gamma=f(\mathbf{c})$, read

$$
\begin{gathered}
\frac{\partial \varepsilon_{i}}{\partial \ln c_{1, j}}=\mathrm{L}_{1, i j}+\sum_{m=1}^{N_{1}} \mathrm{~L}_{1, i m} \frac{\partial \ln \gamma_{1, m j}}{\partial \ln c_{1, j}}+ \\
+\sum_{l=1}^{N_{2}} \mathrm{~L}_{2, i l}\left(\frac{\partial \ln \gamma_{2, l j}}{\partial \ln c_{1, j}}+\frac{\partial \ln c_{2, l j}}{\partial \ln c_{1, j}}\right) \\
i=1, \ldots, N_{a} \\
j=1, \ldots, N_{1}
\end{gathered}
$$

$398 \quad$ Matrices $\mathbf{L}_{1}$ and $\mathbf{L}_{2}$ are the parts of matrix $\mathbf{L}$ relative to primary and 399 secondary species, respectively.

400 The derivatives of (20) with respect to the state variables can be calcu401 lated from 


$$
\begin{aligned}
& \frac{\partial \varepsilon_{i}}{\partial \ln c_{1, j}}=\prod_{n=1}^{N_{s}} a_{n}{ }^{\mathrm{L}_{i n}} \\
& \cdot\left[\mathrm{L}_{1, i j}+\sum_{m=1}^{N_{1}} \mathrm{~L}_{1, i m} \frac{\partial \ln \gamma_{1, m j}}{\partial \ln c_{1, j}}+\sum_{l=1}^{N_{2}} \mathrm{~L}_{2, i l}\left(\frac{\partial \ln \gamma_{2, l j}}{\partial \ln c_{1, j}}+\frac{\partial \ln c_{2, l j}}{\partial \ln c_{1, j}}\right)\right] \\
& i=1, \ldots, N_{a} \\
& j=1, \ldots, N_{1}
\end{aligned}
$$

\section{References}

Appelo, C.A.J., Postma, D., 2010. Geochemistry, groundwater and pollution. Taylor \& Francis.

Back, W., Hanshaw, B.B., Pyle, T.E., Plummer, L.N., Weidie, A., 1979. Geochemical significance of groundwater discharge and carbonate solution to the formation of caleta xel ha, quintana roo, mexico. Water Resources Research 15, 1521-1535.

Ball, J.W., Nordstrom, D.K., 1991. User's manual for WATEQ4F, with revised thermodynamic data base and test cases for calculating speciation of major, trace, and redox elements in natural waters. US Geological Survey Denver, CO.

Bea, S., Carrera, J., Ayora, C., Batlle, F., Saaltink, M., 2009. Cheproo: A fortran 90 object-oriented module to solve chemical processes in earth science models. Computers \& Geosciences 35, 1098-1112. 
Bear, J., 1999. Seawater intrusion in coastal aquifers concepts, methods and practices. Springer.

Bethke, C., 2008. Geochemical and biogeochemical reaction modeling. volume 543. Cambridge University Press Cambridge, UK.

Brassard, P., Bodurtha, P., 2000. A feasible set for chemical speciation problems. Computers \& Geosciences 26, 277-291.

Cabaniss, S.E., 1997. Propagation of Uncertainty in Aqueous Equilibrium Calculations: Non-Gaussian Output Distributions. Analytical Chemistry 69, 3658-3664.

Cabaniss, S.E., 1999. Uncertainty propagation in geochemical calculations: non-linearity in solubility equilibria. Applied Geochemistry 14, 255-262.

Carrayrou, J., Mose, R., Behra, P., 2002. New Efficient Algorithm for Solving Thermodynamic Chemistry. Environmental and Energy Engineering 48, 894-904.

Charlton, S.R., Macklin, C.L., Parkhurst, D., 1997. Phreeqcia graphical user interface for the geochemical computer program phreeqc. US Geological Survey Water-Resources Investigations Report 9.

Criscenti, L.J., Laniak, G.F., Erikson, R.L., 1996. Propagation of uncertainty through geochemical code calculations. Geochimica et Cosmochimica Acta $60,3551-3568$.

De Simoni, M., Carrera, J., Sanchez-Vila, X., Guadagnini, A., 2005. A 
procedure for the solution of multicomponent reactive transport problems. Water resources research 41.

De Simoni, M., Sanchez-Vila, X., Carrera, J., Saaltink, M., 2007. A mixing ratios-based formulation for multicomponent reactive transport. Water Resources Research 43.

Denison, F.H., Garnier-Laplace, J., 2005. The effects of database parameter uncertainty on uranium (vi) equilibrium calculations. Geochimica et cosmochimica acta 69, 2183-2191.

Duan, Z., Li, D., 2008. Coupled phase and aqueous species equilibrium of the $\mathrm{H}_{2} \mathrm{O}-\mathrm{CO}_{2}-\mathrm{NaCl}-\mathrm{CaCO}_{3}$ system from 0 to 250 c, 1 to 1000 bar with nacl concentrations up to saturation of halite. Geochimica et Cosmochimica Acta 72, 5128-5145.

Gupta, H.V., Clark, M.P., Vrugt, J.A., Abramowitz, G., Ye, M., 2012. Towards a comprehensive assessment of model structural adequacy. Water Resources Research 48.

Gustafsson, J.P., 2011. Visual MINTEQ 3.0 user guide. Royal Institute of Technology: Stockholm, Sweden.

Helgeson, H.C., Kirkham, D.H., 1974. Theoretical prediction of the thermodynamic behavior of aqueous electrolytes at high pressures and temperatures; ii, debye-huckel parameters for activity coefficients and relative partial molal properties. American Journal of Science 274, 1199-1261.

Kräutle, S., 2011. The semismooth newton method for multicomponent reactive transport with minerals. Advances in Water Resources 34, 137-151. 
Kräutle, S., Knabner, P., 2005. A new numerical reduction scheme for fully coupled multicomponent transport-reaction problems in porous media. Water resources research 41.

Kulik, D.A., Wagner, T., Dmytrieva, S.V., Kosakowski, G., Hingerl, F.F., Chudnenko, K.V., Berner, U.R., 2013. Gem-selektor geochemical modeling package: revised algorithm and gems3k numerical kernel for coupled simulation codes. Computational Geosciences 17, 1-24.

Mayer, K.U., Frind, E.O., Blowes, D.W., 2002. Multicomponent reactive transport modeling in variably saturated porous media using a generalized formulation for kinetically controlled reactions. Water Resources Research $38,13-1$.

Meeussen, J.C., 2003. Orchestra: An object-oriented framework for implementing chemical equilibrium models. Environmental science \& technology $37,1175-1182$

Merino, E., 1979. Internal consistency of a water analysis and uncertainty of the calculated distribution of aqueous species at $25 \mathrm{c}$. Geochimica et Cosmochimica Acta 43, 1533-1542.

Molins, S., Carrera, J., Ayora, C., Saaltink, M.W., 2004. A formulation for decoupling components in reactive transport problems. Water Resources Research 40.

Nitzsche, O., Meinrath, G., Merkel, B., 2000. Database uncertainty as a limiting factor in reactive transport prognosis. Journal of contaminant Hydrology 44, 223-237. 
Ödegaard-Jensen, A., Ekberg, C., Meinrath, G., 2004. LJUNGSKILE: a program for assessing uncertainties in speciation calculations. Talanta 63, 907-916.

Parkhurst, D.L., Appelo, C., et al., 1999. User's guide to PHREEQC (Version 2): A computer program for speciation, batch-reaction, one-dimensional transport, and inverse geochemical calculations. US Geological Survey Denver.

Paz-García, J.M., Johannessona, B., Ottosena, L.M., Ribeirob, A.B., Rodríguez-Marotoc, J.M., 2013. Computing multi-species chemical equilibrium with an algorithm based on the reaction extents. Computers \& Chemical Engineering .

Rezaei, M., Sanz, E., Raeisi, E., Ayora, C., Vázquez-Suñé, E., Carrera, J., 2005. Reactive transport modeling of calcite dissolution in the fresh-salt water mixing zone. Journal of Hydrology 311, 282-298.

Saaltink, M.W., Ayora, C., Carrera, J., 1998. A mathematical formulation for reactive transport that eliminates mineral concentrations. Water Resources Research 34, 1649-1656.

Saaltink, M.W., Vilarrasa, V., De Gaspari, F., Silva, O., Carrera, J., Rötting, T.S., 2013. A method for incorporating equilibrium chemical reactions into multiphase flow models for $\mathrm{CO}_{2}$ storage. Advances in Water Resources 62, $431-441$.

Sanz, E., Ayora, C., Carrera, J., 2011. Calcite dissolution by mixing waters: 
geochemical modeling and flow-through experiments. Geologica Acta 9, $67-77$.

Smith, S.D., Adams, N.W.H., Kramer, J.R., 1999. Resolving uncertainty in chemical speciation determinations. Geochimica et Cosmochimica Acta $63,3337-3347$.

Steefel, C., Yabusaki, S.B., 2000. OS3D/GIMRT software for modeling multicomponent-multidimensional reactive transport. Technical Report. Pacific Northwest National Lab., Richland, WA (US).

Weber, C.L., VanBriesen, J.M., Small, M.S., 2006. A stochastic regression approach to analyzing thermodynamic uncertainty in chemical speciation modeling. Environmental science \& technology 40, 3872-3878.

Werner, A.D., Bakker, M., Post, V.E., Vandenbohede, A., Lu, C., AtaieAshtiani, B., Simmons, C.T., Barry, D.A., 2013. Seawater intrusion processes, investigation and management: Recent advances and future challenges. Advances in Water Resources 51, 3-26.

Wolery, T.J., 1983. EQ3NR: a computer program for geochemical aqueous speciation-solubility calculations. Users guide and documentation. Technical Report. Lawrence Livermore National Lab., CA (United States).

Wolery, T.J., 1992. EQ3NR, a Computer Program for Geochemical Aqueous Speciation-solubility Calculations: Theoretical Manual, User's Guide and Related Documentation (Version 7.0). Lawrence Livermore Laboratory, University of California. 
${ }_{527}$ Xu, T., Sonnenthal, E., Spycher, N., Pruess, K., 2006. TOUGHREACT ${ }_{528}$ A simulation program for non-isothermal multiphase reactive geochemical 529 transport in variably saturated geologic media: Applications to geothermal 530 injectivity and $\mathrm{CO}_{2}$ geological sequestration. Computers \& Geosciences 32, $531 \quad 145-165$. 


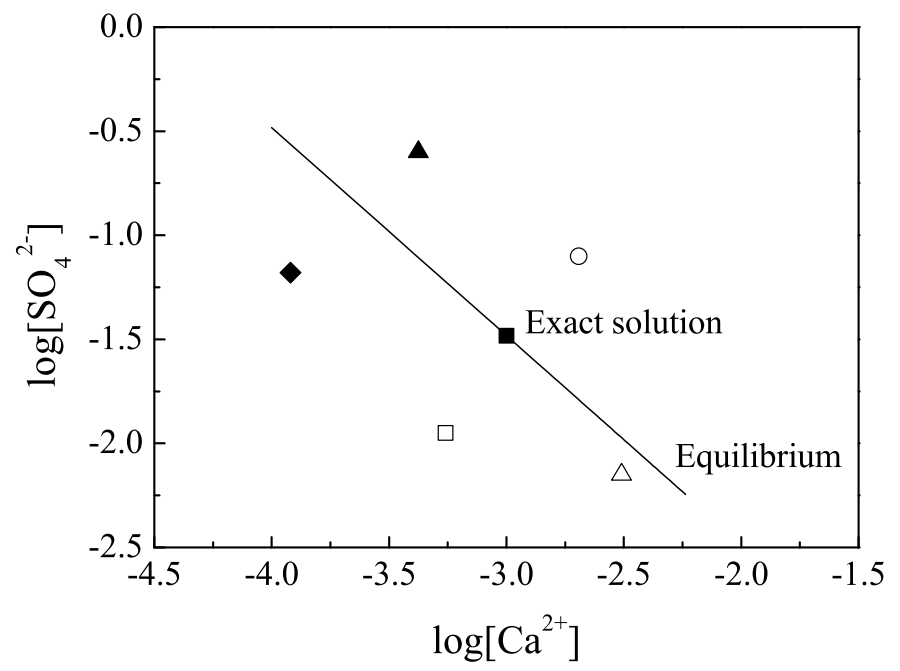

Figure 1: Five measurements, exact solution and equilibrium line for gypsum example.

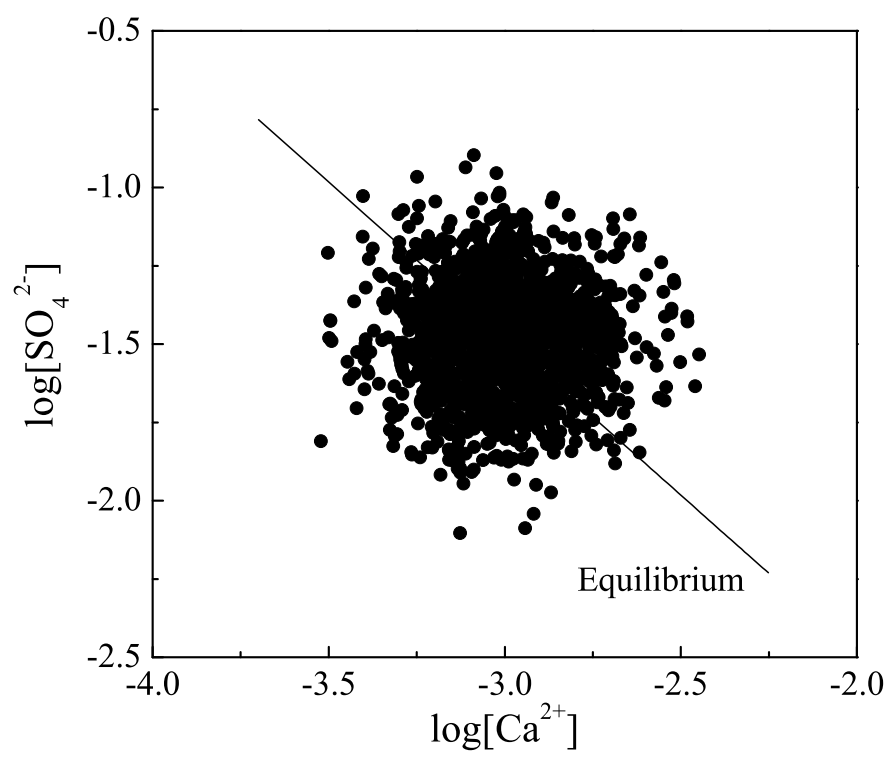

Figure 2: 1500 measurement points generated by means of a lognormal distribution for gypsum example. 


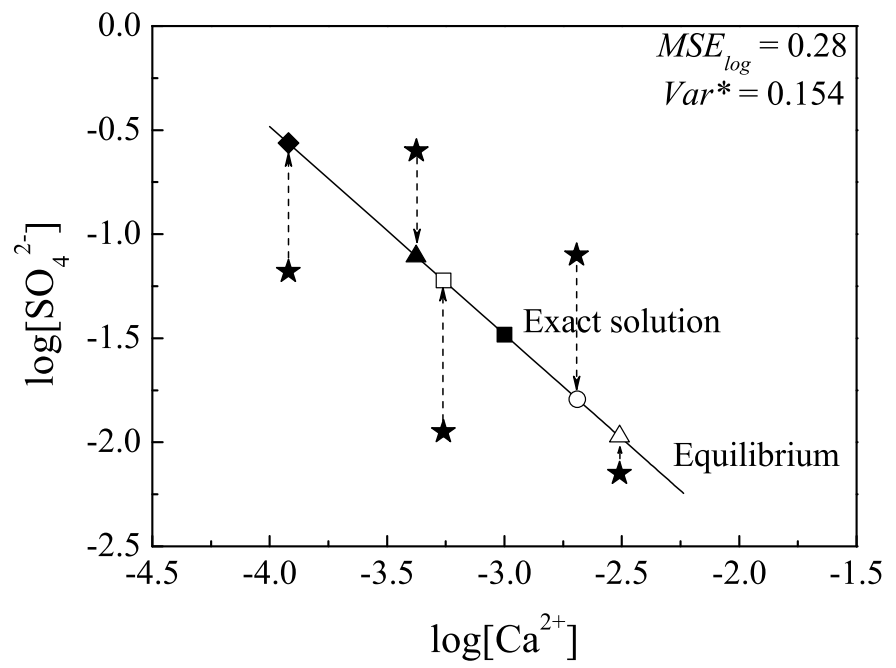

Figure 3: Speciation results of traditional speciation method, exact solution and equilibrium line for gypsum example. Dashed arrows show the movement of the five points from initial conditions, represented with stars.

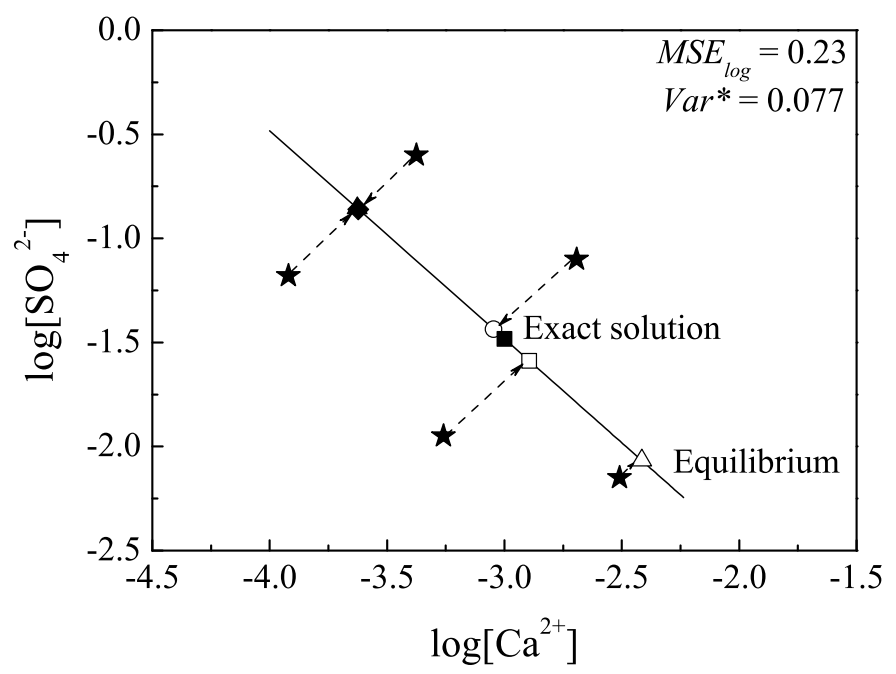

Figure 4: Speciation results of proposed method, exact solution and equilibrium line for gypsum example. Dashed arrows show the movement of the five points from initial conditions, represented with stars. 


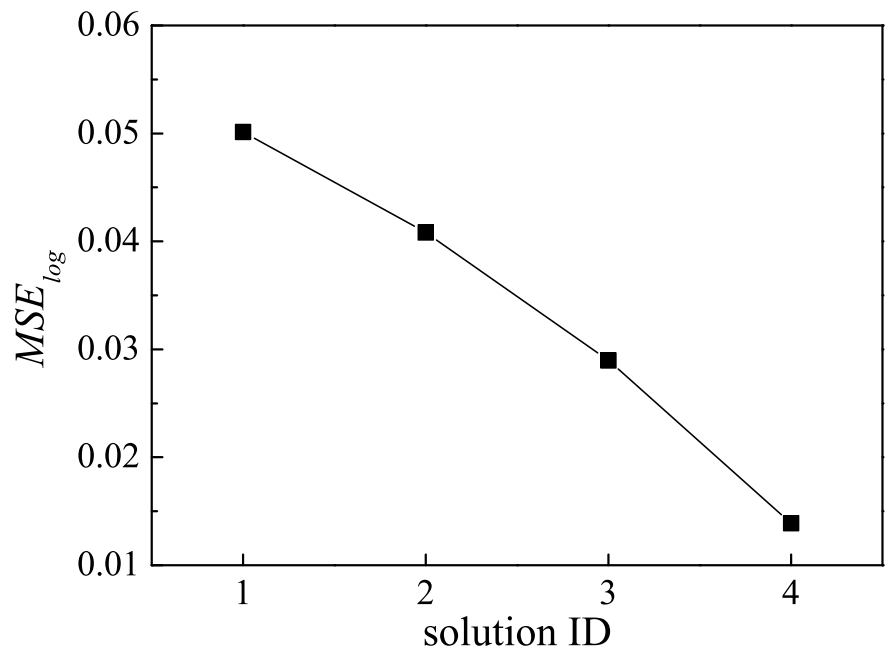

Figure 5: $M S E_{\text {log }}$ for traditional speciation method (solution 1) and proposed method (solutions 2, 3 and 4) obtained with $\sigma_{e}$ values of table 2 . 

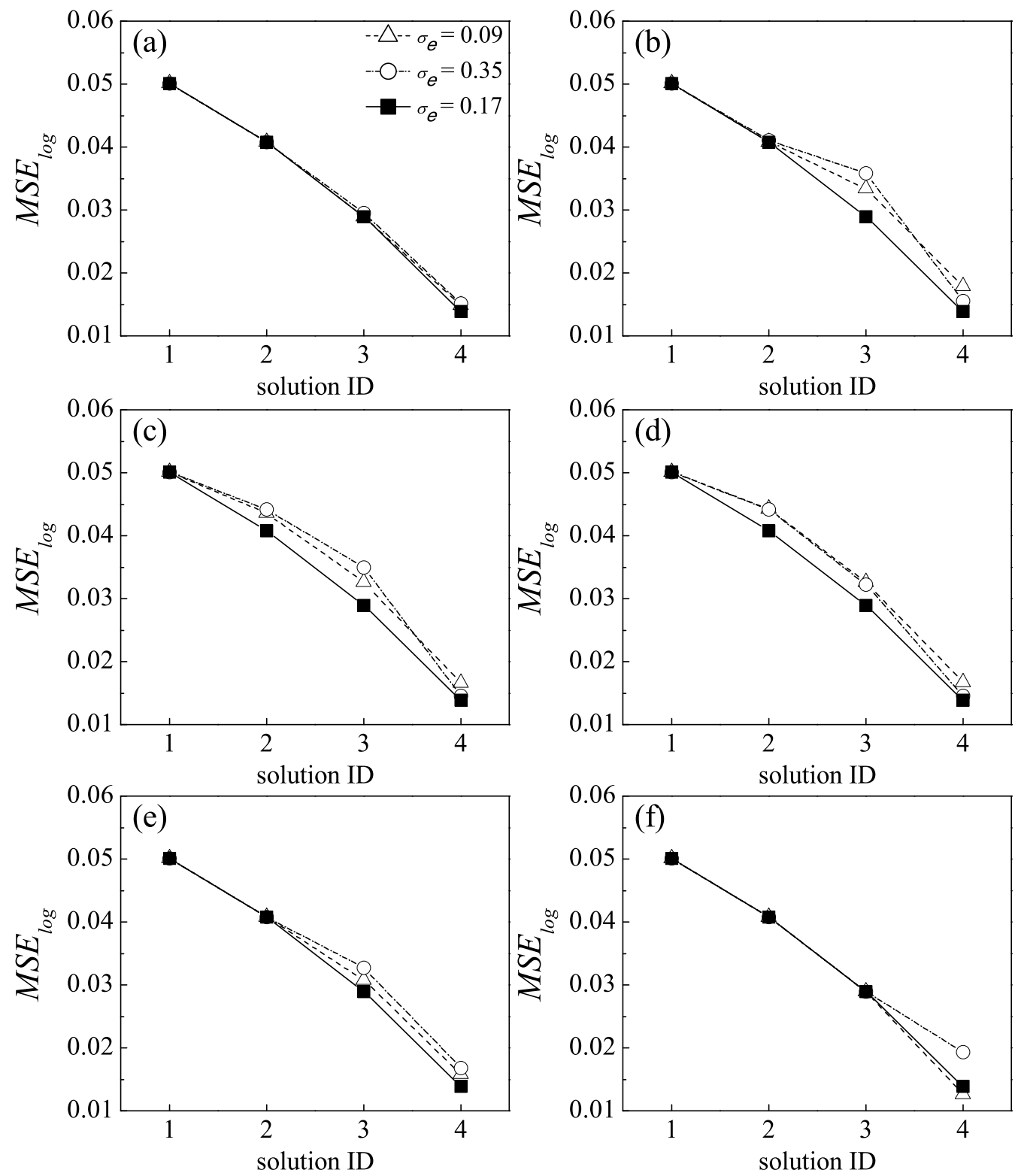

Figure 6: $M S E_{\log }$ obtained changing values of $\sigma_{e}$ of each data: (a) $C a_{t o t}$; (b) $\mathrm{a}_{H^{+}}$; (c) alkalinity; (d) $T I C$; (e) $\mathrm{a}_{C O_{2(g)}}$; (f) calcite equilibrium. 

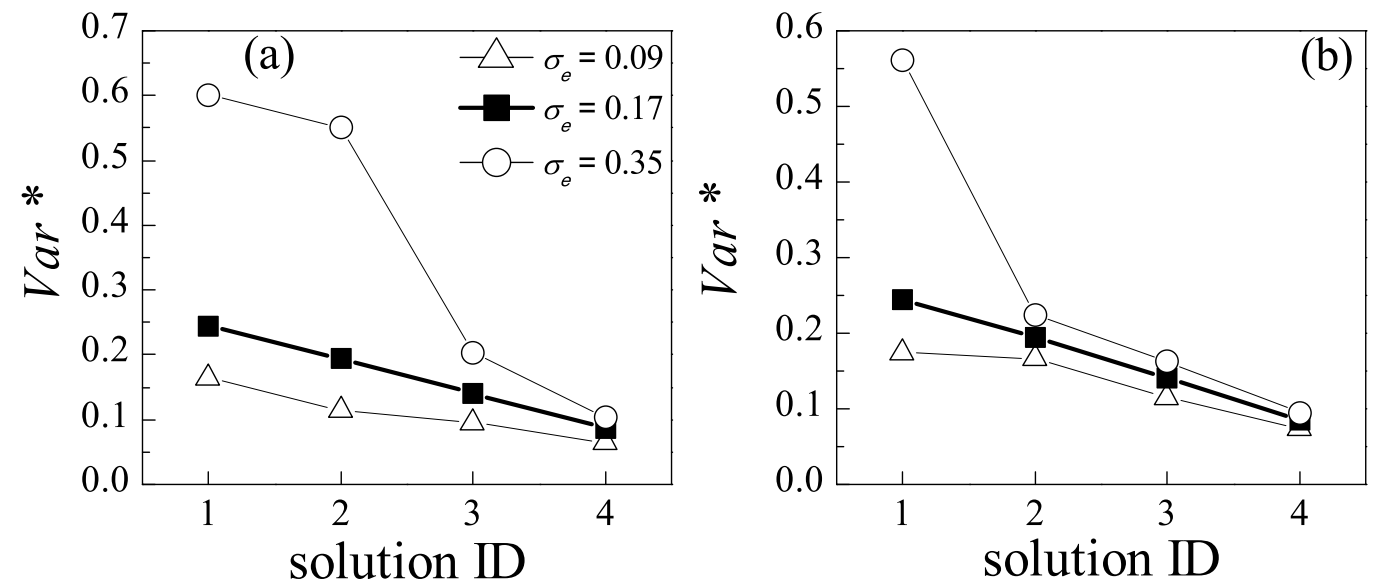

Figure 7: $\operatorname{Var}^{*}$ obtained for different values of $\sigma_{e}$ of (a) $\mathrm{a}_{H^{+}}$and (b) alkalinity. 
Table 1: Exact solution for carbonate example.

\begin{tabular}{ccc}
\hline & Species & Values \\
\hline$c^{*}$ & $\mathrm{Ca}^{2+}$ & $4.92 \cdot 10^{-4}$ \\
{$[\mathrm{~mol} / \mathrm{l}]$} & $\mathrm{H}^{+}$ & $5.51 \cdot 10^{-9}$ \\
& $\mathrm{HCO}_{3}^{-}$ & $9.63 \cdot 10^{-4}$ \\
& $\mathrm{CO}_{2(a q)}$ & $1.07 \cdot 10^{-5}$ \\
& $\mathrm{CO}_{3}^{2-}$ & $9.78 \cdot 10^{-6}$ \\
& $\mathrm{OH}^{-}$ & $2.01 \cdot 10^{-6}$ \\
\hline $\mathrm{a}^{*}$ & $\mathrm{CO}_{2(g)}$ & $3.16 \cdot 10^{-4}$ \\
{$[$ bar $]$} & & \\
\hline SI & Calcite & 0.0
\end{tabular}

(Sat. Index) 
Table 2: Mean values and standard deviations adopted to generate 1500 realizations of data for the carbonate example.

\begin{tabular}{ccccc}
\hline Data & $\mu$ & $\sigma_{g}$ & $\sigma_{e}$ & $\varepsilon$ equation \\
\hline$C a_{\text {tot }}$ & $4.92 \cdot 10^{-4}$ & 0.17 & 0.17 & $(21)$ \\
$\mathrm{a}_{H^{+}}$ & $5.29 \cdot 10^{-9}$ & 0.17 & 0.17 & $(22)$ \\
Alkalinity & $9.85 \cdot 10^{-4}$ & 0.17 & 0.17 & $(21)$ \\
TIC & $9.84 \cdot 10^{-4}$ & 0.17 & 0.17 & $(21)$ \\
$\mathrm{a}_{C O_{2(g)}}$ & $3.16 \cdot 10^{-4}$ & 0.17 & 0.17 & $(22)$ \\
Calcite Eq. & 0 & 0 & 0.17 & $(22)$ \\
\hline
\end{tabular}

Units are in $m o l / l$ except for $\mathrm{a}_{\mathrm{CO}_{2(g)}}$ which is expressed in bar. 